J. Dairy Sci. 92:2991-3001

doi:10.3168/jds.2008-1642

(C) American Dairy Science Association, 2009.

\title{
Variations in bovine milk oligosaccharides during early and middle lactation stages analyzed by high-performance liquid chromatography-chip/mass spectrometry
}

\author{
N. Tao, ${ }^{*}$ E. J. DePeters,† J. B. German,‡ R. Grimm,§ and C. B. Lebrilla*1 \\ *Department of Chemistry and Biochemistry (School of Medicine), \\ †Department of Animal Science, and \\ ‡Department of Food Science and Technology, University of California, Davis 95616 \\ §Agilent Technologies Inc., Santa Clara, CA 95052
}

\section{ABSTRACT}

Milk oligosaccharides (OS) are not only a source of nutrition for newborns, but also provide numerous important biological functions including the prevention of pathogen binding to the intestinal epithelium and serving as nutritive sources for beneficial bacteria. High-performance mass spectrometry and separation methods were used to evaluate changes of bovine milk oligosaccharides (bMO) in different lactation stages. Previously, 40 bMO were identified in bovine milk with many consisting of short oligomeric chains that were less complex than human milk oligosaccharides (hMO). The bMO are also significantly more anionic than hMO, with nearly $70 \%$ in measured abundances containing either $\mathrm{N}$-acetylneuraminic acid or $\mathrm{N}$-glycolylneuraminic acid, and no fucosylated OS. In this study, we examined factors that could affect the abundances of bMO including stage of lactation and breed. The total concentrations dropped rapidly in the first several days of lactation. Moreover, the anionic oligosaccharides (including $N$-glycolylneuraminic acid) decreased more rapidly compared with the neutral oligosaccharides.

Key words: oligosaccharide, bovine milk, mass spectrometry

\section{INTRODUCTION}

Milk is a complex food made up of components such as lipids, oligosaccharides (OS), proteins, amino acids, vitamins, minerals, and other bioactive peptides (Jackson et al., 1995). As the third most abundant component after lipids and lactose, human milk oligosaccharides (hMO) have been widely recognized for their contribution to the establishment of intestinal flora by stimulating the growth of beneficial bacteria and acting

Received August 19, 2008.

Accepted February 18, 2009.

${ }^{1}$ Corresponding author: cblebrilla@ucdavis.edu as free receptors that bind to pathogenic organisms in the gastrointestinal tract of infants (Newburg, 1996; Kunz et al., 2000; Newburg et al., 2005; Boehm and Stahl, 2007).

Bovine milk is a common ingredient in infant formula as well as a basic material for the dairy and food industries. Furthermore, OS from bovine milk could be a premier choice of a reasonable source of nutrition with biological functions close to those of hMO. Comprehensive studies have attempted to characterize the complexity of hMO (Newburg, 2000; Pfenninger et al., 2001; Newburg et al., 2004; Ninonuevo et al., 2006; German et al., 2008), but little research has been done on bovine milk oligosaccharides (bMO) (Gopal and Gill, 2000; Urashima et al., 2001; Martin-Sosa et al., 2003). Bovine milk contains significantly less free OS compared with human milk (Boehm et al., 2005; Brunser et al., 2006; Bongers et al., 2007). Concentrations of hMO range between 20 and $23 \mathrm{~g} / \mathrm{L}$ in colostrum (Coppa et al., 1999) and 7 to $12 \mathrm{~g} / \mathrm{L}$ in mature milk (Boehm and Stahl, 2007), whereas concentrations of bMO in colostrum are approximately 20-fold lower (Ali-Vehmas et al., 2001), which make it more challenging to profile bMO qualitatively as well as quantitively.

Because of the complexity and lower amount of bMO, analysis of bMO has been hindered by the lack of effective analytical tools. The major analytical techniques currently include nuclear magnetic resonance (NMR) spectroscopy (Guerardel et al., 1999), mass spectrometry (Pfenninger et al., 2001, 2002), and chromatography such as high $\mathrm{pH}$ anion-exchange chromatography with pulsed amperometric detection (Kunz and Rudloff, 1996), capillary electrophoresis, and HPLC (Martin-Sosa et al., 2003). We have recently reported the characterization of hMO by using nanoflow liquid chromatography (nanoLC) and high-performance MS (Ninonuevo et al., 2006). The integration of nanoLC with MS in a HPLC-chip/time-of-flight MS (HPLCchip/TOF MS) instrument allowed the rapid profiling of $\mathrm{hMO}$ and $\mathrm{bMO}$ in a precise and reproducible 
manner. With this system, we were able to identify 40 $\mathrm{bMO}$, most of which were in the sialylated forms (Tao et al., 2008), which doubles the number of total published bMO. Moreover, sialic acids have been reported to be essential for infant brain development and protection by binding to pathogens in the gut. The high content of anionic (sialylated) OS makes bovine milk a potentially valuable source of sialic acids. In contrast, fucosylation, which is found in $70 \%$ of hMO, is not observed at any appreciable level in bovine milk.

Several studies have reported the composition, structure, and some bioactivities of hMO, but only a small number of publications have examined changes during lactation (Newburg, 2000; Chaturvedi et al., 2001; Coppa et al., 2001; Suzuki and Suzuki, 2001; Boehm and Stahl, 2007; Ninonuevo et al., 2008). Fewer studies are reported for the variations study in bMO during the long lactation progression of bovines (Martin-Sosa et al., 2003; McJarrow and van Amelsfort-Schoonbeek, 2004). McJarrow and van Amelsfort-Schoonbeek (2004) described seasonal variations in the concentrations of bMO in Jersey and Friesian cows but monitored only 4 OS species over the first $5 \mathrm{~d}$ of milking. They noted significant differences between structures and relative abundances in different breeds. Martin-Sosa's group was able to monitor 5 sialylated OS from samples collected during the first month (Martin-Sosa et al., 2003). These studies demonstrate the challenge in getting detailed information about OS changes at the stages of lactation. In this study, we used nanoLC separation and high-performance MS as a quantitative tool for monitoring changes in composition and abundance of bMO during the early and middle lactation periods. Moreover, we examined variation between Holstein and Jersey cows and compared sialylation levels in a small group.

\section{MATERIALS AND METHODS}

\section{Materials}

Nonporous graphitized carbon cartridges (150 mg of bed weight, $4 \mathrm{~mL}$ tube size) were obtained from Alltech (Deerfield, IL). Sodium borohydride (98\%) and 2,5-dihydroxybenzoic acid were purchased from SigmaAldrich (St. Louis, MO). All reagents were of analytical or HPLC grade.

\section{Collection of Milk Samples}

Thirty-nine postpartum colostrum and mature milk samples from individual Jersey and Holstein cows $(\mathrm{n}=$ 13) were collected within $12 \mathrm{~h}$ of calving, at d 6 , and at d 120 of lactation. Milk samples were frozen at $-80^{\circ} \mathrm{C}$ after collection. Individual milk samples were analyzed with mass spectrometry.

\section{Sample Preparation}

Milk samples were completely thawed, and $0.5 \mathrm{~mL}$ was centrifuged at $4,000 \times g$ in a microfuge for $30 \mathrm{~min}$ at $4^{\circ} \mathrm{C}$. After the top fat layer was removed, 4 volumes of chloroform/methanol $(2: 1, \mathrm{vol} / \mathrm{vol})$ and 2 volumes of deionized $\mathrm{H}_{2} \mathrm{O}$ were added to the defatted milk samples. After centrifugation at $4,000 \times g$ for $30 \mathrm{~min}$ at $4^{\circ} \mathrm{C}$, the upper layer was carefully transferred. Two volumes of ethanol were added to the mixture overnight at $4^{\circ} \mathrm{C}$, followed by a 30-min centrifugation at $4,000 \times$ $g$ and $4^{\circ} \mathrm{C}$ to remove denatured protein. The supernatant (milk OS-rich fraction) was freeze-dried using a speed vacuum. Native milk OS were reduced to alditol forms by using $1.0 \mathrm{M}$ sodium borohydride in $\mathrm{H}_{2} \mathrm{O}$ and incubated at $42^{\circ} \mathrm{C} 17 \mathrm{~h}$. The bMO were purified by solid-phase extraction using a nonporous graphitized carbon cartridge and eluted with $20 \%$ acetonitrile in water (vol/vol) before MS analysis.

\section{HPLC-Chip/TOF MS}

Milk OS fractions collected after solid-phase extraction with the graphitized carbon cartridge were analyzed using a microfluidic 6200 Series HPLC-chip/TOF MS instrument (Agilent Technologies, Santa Clara, CA). The microfluidic HPLC-chip consists of an enrichment column, a liquid chromatography separation column packed with porous graphitized carbon, and a nanoelectrospray tip. Separation was performed by using a binary gradient: $\mathrm{A}=3 \%$ acetonitrile in $0.1 \%$ formic acid solution and $\mathrm{B}=90 \%$ acetonitrile in $0.1 \%$ formic acid solution. The column was initially equilibrated and eluted at a flow rate of $0.3 \mu \mathrm{L}$ for nanopump and 4 $\mu \mathrm{L}$ for capillary pump. The 65 -min gradient was programmed as follows: 2.5 to $20 \mathrm{~min}, 0-16 \% \mathrm{~B} ; 20$ to 30 min, 16-44\% B; 30 to 35 min, B increased to $100 \%$, and continued with $100 \%$ B to 45 min, and finally, $0 \%$ B for 20 min to equilibrate the chip column before the next sample injection. Each composition of milk OS was identified with an in-house program (Glycan Finder). Distinct species were identified based on accurate mass and reproducible retention times. The instrument performed automatic auto-tuning using a dual nebulizer electrospray source with an automated internal calibrant consisting of an unknown fluorinated compound delivery system, which introduced a constant flow (100 $\mu \mathrm{L} / \mathrm{min}$ ) of calibrating solution containing the internal reference masses $(m / z$ 118.0863, 322.0481, 622.0290, 922.0098, 221.9906, 1521.9715, 1821.9523, 2121.9332, 2421.9140, and 2721.8948) in positive mode. 


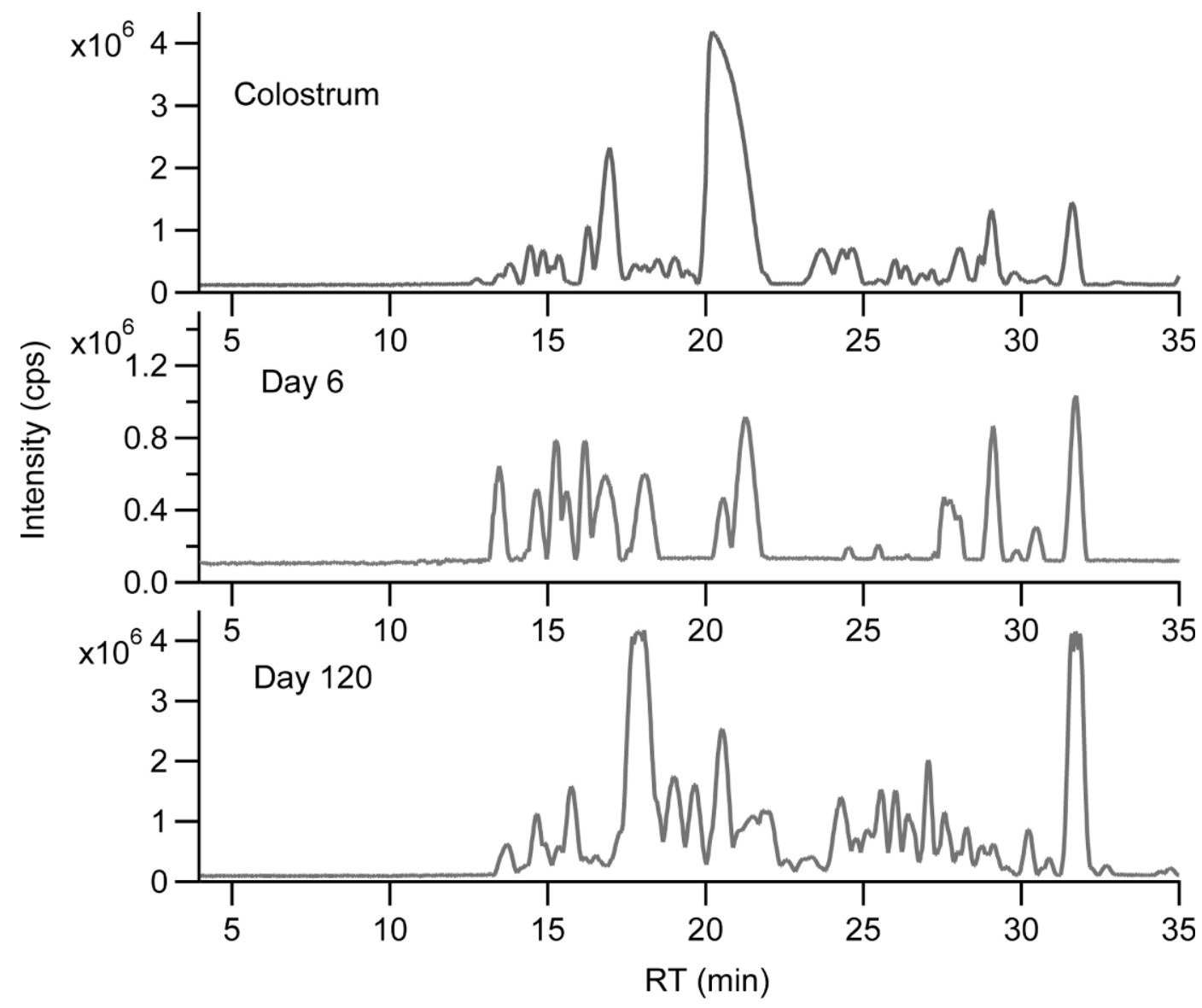

Figure 1. High-performance liquid chromatography-chip/time-of-flight-MS chromatograms of bovine milk oligosaccharides of colostrum (d 1), d 6, and d 120 milk from Holstein cow 1950. RT = retention time.

\section{RESULTS AND DISCUSSION}

Forty OS species were previously identified employing nano-electrospray ionization Fourier transform ion cyclotron resonance (nESI-FTICR) and HPLC-chip/ TOF MS (Tao et al., 2008). Complete and partial structures were accomplished for most of the OS using the tandem MS/MS technique called infrared multiphoton dissociation (IRMPD; Tao et al., 2008). Knowledge of these structures, their unique retention times, and accurate masses aided in monitoring specific OS species for changes in abundances. Quantitation in each case was performed based strictly on the measured abundances in the HPLC-chip/TOF MS.

\section{Variations in Holstein Colostrum, d 6 Milk, and d 120 Milk OS}

The chromatograms obtained with the nanoLC-Chip/ TOF MS for milk samples collected from colostrums, d 6 milk, and d 120 milk after calving for an individual Holstein cow are shown in Figure 1. Oligosaccharide compositions were determined based on mass accuracy of less than $5 \mathrm{ppm}$. Detailed information about profiling bMO compositions and structures is provided in our previously published work (Tao et al., 2008). The major components in bMO are anionic OS, in contrast to the neutral OS found in human milk. Moreover, fucosylated OS are absent in bMO, whereas they comprise more than $70 \%$ of the OS in hMO. Interestingly, fucosylated protein bound OS were found predominantly on bovine milk fat globule membrane protein (Wilson et al., 2008). All ions in the positive mode corresponded to protonated or ammonium-coordinated species in the form of $[\mathrm{M}+\mathrm{H}]^{+}$or $\left[\mathrm{M}+\mathrm{NH}_{4}\right]^{+}$, respectively. The predominant peak shown in the colostrum chromatogram with a retention time around 20 to 21 min corresponds to 3 '-sialyllactose [NeuAc( $\alpha 2-3) \mathrm{Gal}(\beta 1-4) \mathrm{Glc}]$. Decreases in concentration in bovine milk after parturition have been shown. The peak sharply decreased to d 6 and continued to decrease to d 120. From the base peak chromatogram, it is sometimes difficult to visualize quantities because oligomers with similar structure but different sizes often overlap; isomers tend to separate 

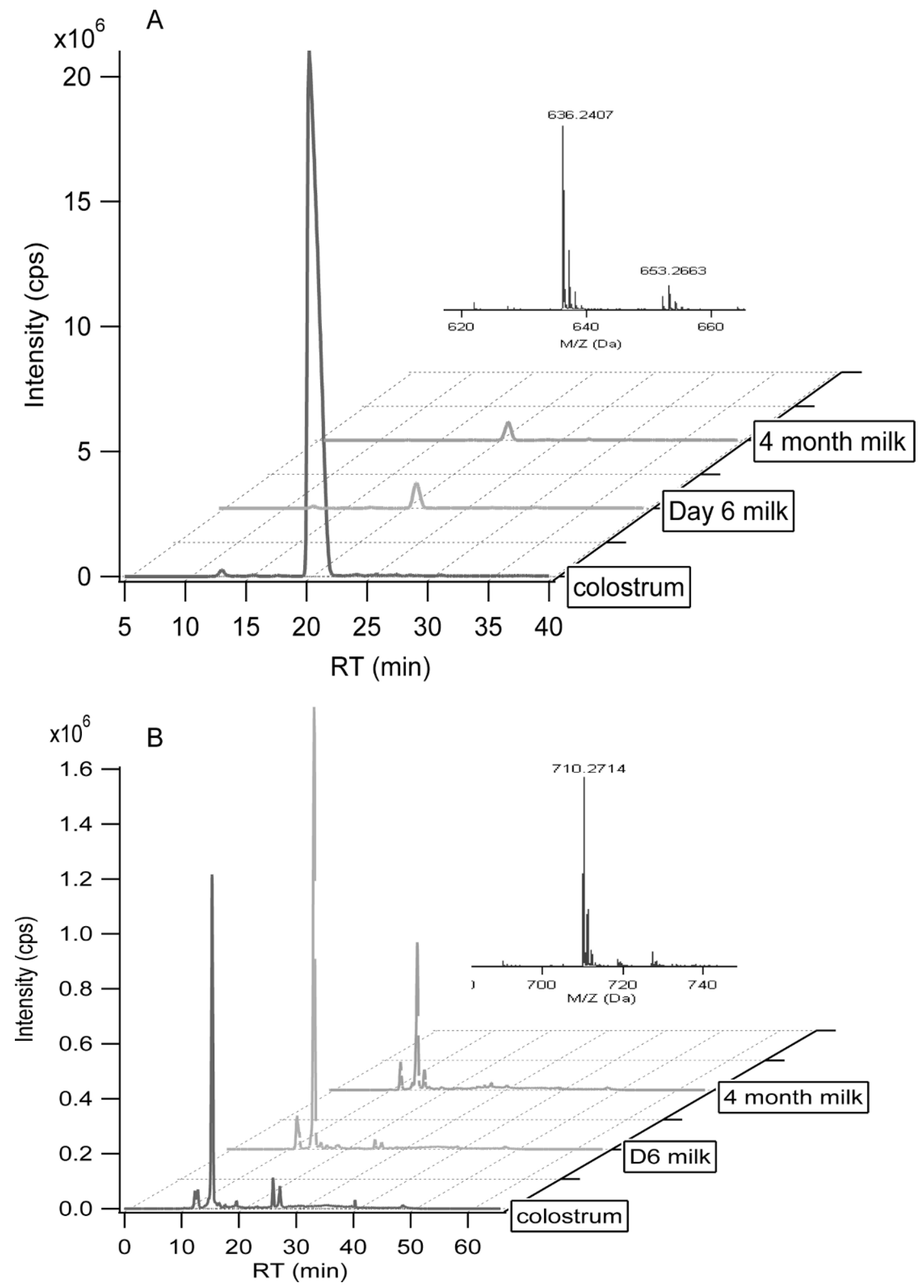

Figure 2. Extracted ion chromatograms of selected ions (A) $m / z 636.2346$ and (B) $m / z 710.2714$ from colostrum (d 1) of Holstein cow 1950 with mass spectra inset. $\mathrm{RT}=$ retention time. 


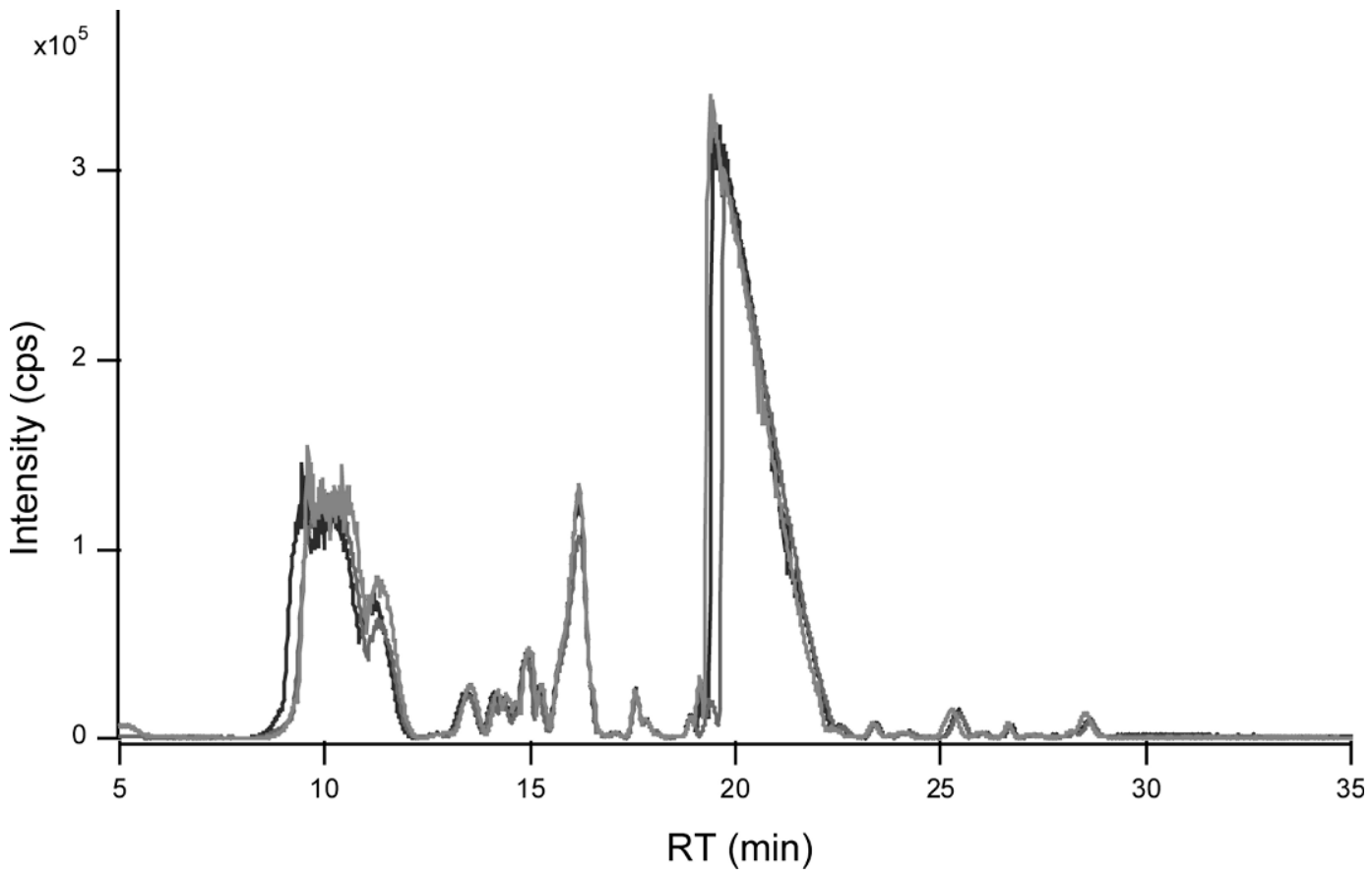

Figure 3. High-performance liquid chromatography-chip/time-of-flight chromatograms of bovine colostrum oligosaccharides from Jersey cows (3 runs overlapped). RT $=$ retention time.

more cleanly. The extracted ion chromatogram is more useful for monitoring specific masses and the associated isomers.

Figure 2 shows the chromatograms of selected ions corresponding to 3 '-sialyllactose $[\operatorname{NeuAc}(\alpha 2-3)$ Gal $(\beta 1-4)$ Glc; Figure $2 \mathrm{~A}]$ and lacto-N-neotetraose [LNnT; $\operatorname{Gal}(\beta 1-4) \operatorname{GlcNAc}(\beta 1-3) \operatorname{Gal}(\beta 1-4)$ Glc; Figure $2 \mathrm{~B}$ ], which represent the major anionic and neutral OS species, respectively, at the 3 lactation time points. 3 '-Sialyllactose is the most abundant of all the bMO; it decreased dramatically in the transitional milk stage (d 6) and progressively decreased in mature milk up to d 120. This trend has been found in most of the other anionic OS species. The mass spectrum is shown in the inset in Figure 2A for $m / z 636.2407$ as the protonated species and $m / z 653.266$ as the ammonium-coordinated species. These changes agree with previous observations using other techniques including high $\mathrm{pH}$ anionexchange chromatography (Nakamura et al., 2003). In contrast, the example representing the neutral OS LNnT increased slightly to $\mathrm{d} 6$ and decreased by d 120 (Figure 2B). These observations agree with the earlier report by Nakamura et al. (2003), which showed that concentrations of anionic OS in bovine milk decreased dramatically after birth, whereas concentrations of total neutral OS increased. However, that study was based on early-lactation data (first $7 \mathrm{~d}$ of milking) and included only the 3 most abundant species that were able to be monitored, all of which were anionic.
High-performance liquid chromatography-chip/ MS is a robust tool to profile bMO at various points during lactation, providing accurate masses, precise compositions, and exact retention time tags with great reproducibility. The chromatographic reproducibility of HPLC-chip/TOF-MS was examined by triple injections of the same sample on a 250-h-old chip (Figure 3 ). The shift of retention time of major peaks is within 0.2 min. The performance is outstanding and the analytical method is very reliable. To improve consistency, all of the samples were analyzed using the same microfluidic chip to avoid any individual variation. Figure 4 shows base peak chromatograms for the milk of 3 Holstein cows. Each chromatogram was plotted on the same fixed time scale for better illustration. Fewer variations in composition and concentration were found in bMO compared with hMO. Furthermore, consistent changes through the progression of lactation stages were found in all the cows examined. Sialyllactose was extremely abundant in all milk samples. The diversity of hMO from individual donors due to genotype, area, diet, and age has been well described (Ninonuevo et al., 2006).

Colostrum and d 6 milk samples were collected from 9 individual Holstein cows, and d 120 milk samples were obtained from 4 Holstein cows to test the transition of oligosaccharide intensity. Figure 5 lists the comparisons among colostrum, d 6, and d 120 samples on relative abundances of total OS, total sialic acid, and N-glycolylneuraminic acid (NeuGc)-containing OS. Values 


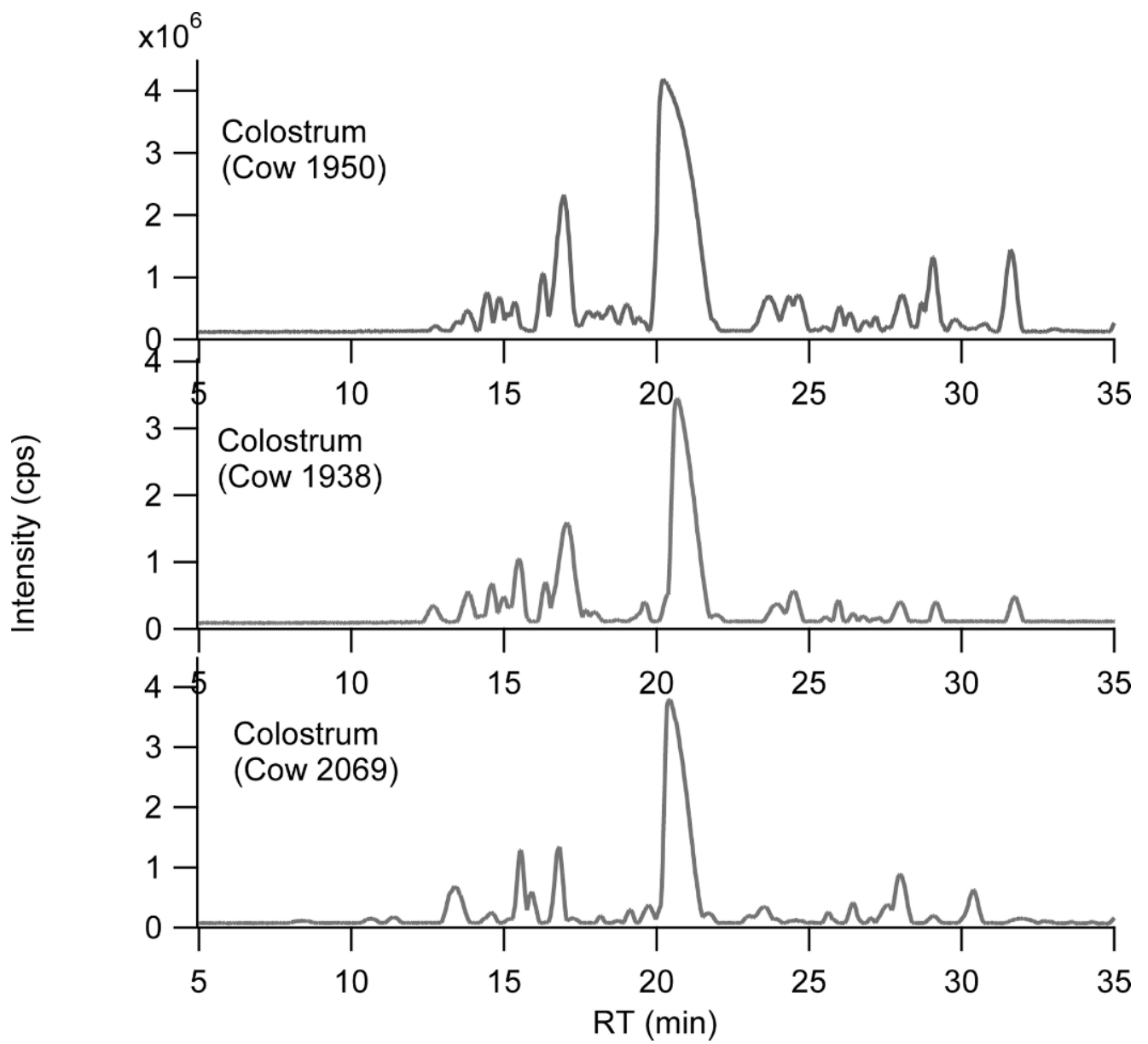

Figure 4. High-performance liquid chromatography-chip/time-of-flight chromatograms of bovine colostrum oligosaccharides from Holstein cow 1950, cow 1938, and cow 2069. RT = retention time.

are means \pm standard deviation $(\mathrm{SD})$ in abundances, with $\mathrm{n}=9$ for colostrum and $\mathrm{d} 6$ milk samples, and $\mathrm{n}$ $=4$ for $\mathrm{d} 120$ milk samples. The individuals have much less diversity compared with human donors. All bMO have the same pattern from colostrum to mature milk. Numbers of OS identified vary from 30 to 40 with the stages of lactation. In bovine milk, sialylation is particularly high in colostrum with over $70 \%$ in abundances, progressively dropping to around $50 \%$ in mature milk; $50 \%$ sialylation is still higher than the level observed in human milk colostrum or mature milk, in which only 10 to $15 \%$ of all OS are sialylated. The anionic OS in cow milk include $N$-acetyl neuraminic acid (NeuAc)linked and NeuGc-linked OS, whereas in human milk, only NeuAc-linked OS exist. The change in sialic acids could be produced in part by the action of bacterial enzymes on sialoglycoconjugates. Furthermore, evidence exists that sialic acids can promote early brain development in young piglets (who have similar brain structure and function as human neonates; Moughan et al., 1992; Wang et al., 2007). Changes in OS types in milk may be relevant to the health of infants consuming bovine milk products because the high concentration of sialylated OS might be important for protecting newborns from pathogens. However, this decrease in the sialic acids is more evident in the first few days after birth, maybe because after some time has elapsed, other mechanisms of the immune system are ready and can guarantee protection to the newborn. In contrast, other neutral OS increased after colostrum, suggesting that the mechanism by which these neutral OS can promote health is by feeding colonic bacteria, acting as prebiotics. Comparison of NeuGc is also shown in Figure 5 (enlarged inset figure) at a low abundance around $5 \%$ in colostrum, decreasing to trace amounts in transitional and mature milk.

The abundance (\%) of OS species from the milk of one typical cow (cow 1950) are presented in Table 1 for samples from the 3 lactation periods. Oligosaccharide species were identified in colostrum, milk at d 6 , and 


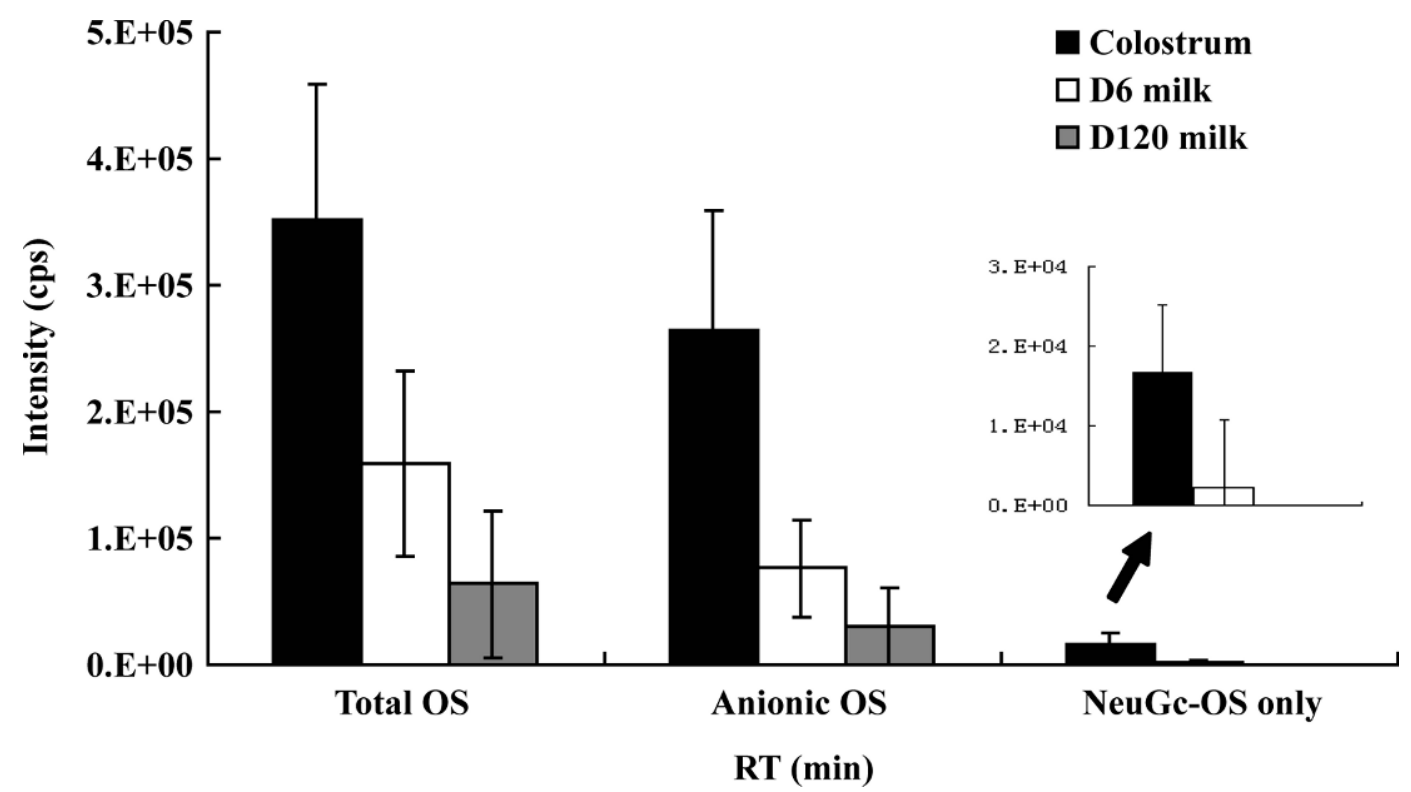

Figure 5. Changes in the total oligosaccharides (OS), total anionic OS, and $N$-glycolylneuraminic acid (NeuGc)-containing OS only content of bovine milk at different stages of lactation (colostrum, d 6, and d 120 representing colostrum, transitional, and mature milk, respectively). Values $($ mean $\pm \mathrm{SD}$ ) are $\mathrm{n}=9$ for colostrum and $\mathrm{d} 6$, and $\mathrm{n}=4$ for $\mathrm{d} 120$ milk samples. $\mathrm{RT}=$ retention time.

milk at d 120 of lactation. The most abundant peak, representing approximately $30 \%$ of total OS, was sialyllactose, with a retention time of $20.5 \mathrm{~min}$. The retention time did not vary significantly between the 3 lactation periods. This peak was identified as OS 13 in Table 1. However, LNnT (OS 5 in Table 1), with a retention time of $15.3 \mathrm{~min}$, increased in d 6 milk and then decreased in the d 120 milk sample. The increase in LNnT from colostrum to d 6 milk might be important for human health as it relates to the postnatal formation of bifidus flora in the infant colon. Lacto-N-tetraose and LNnT were identified in our previous study to be a bifidobacterium growth-stimulating factor, a potential prebiotic (Ward et al., 2006).

Seven OS with NeuGc residues (from 3 to 6 ) were found in bovine colostrum, whereas only 2 were observed in milk at d 6 [composition: 2 hexose $(\mathrm{Hex})+1$ NeuGc and 4 Hex $+1 \mathrm{~N}$-acetylhexosamine (HexNAc) +1 NeuGc). These 2 OS, to the best of our knowledge, were identified for the first time by our group. Interestingly, NeuGc OS were not present or present only in trace amounts in d 120 milk. For the NeuAc-containing OS, 23 were identified in colostrum, and only 4 of these OS were missing in d 6 milk. Furthermore, 11 OS were found in d 120 milk. Three milk samples from cow 1950 show that NeuAc-linked OS reached a value of $73 \%$ in colostrum, and neutral OS components accounted for $22 \%$ of the total OS. In addition, NeuGc OS accounted for approximately $5 \%$ in colostrum. The anionic OS components dropped dramatically in d 6 milk, where
NeuAc comprised $53.7 \%$ and NeuGc decreased to $3.3 \%$. Neutral OS increased to $43 \%$, a doubling of its concentration. Compared with OS in d 6 milk and colostrum, d 120 milk OS were very similar to d 6 bMO. Milk from d 120 contained 54.9\% NeuAc-linked OS, $45 \%$ neutral OS, and no NeuGc-linked OS. Furthermore, the abundances of each type of OS in d 120 milk were slightly lower than those of d 6 milk samples. Total abundance of OS in colostrum was highest for the 3 lactation periods, and decreased to $30 \%$ in d 6 milk (data not shown). All of the acidic OS, the major component of bMO, significantly decreased, confirming the data reported by Urashima's group (Nakamura et al., 2003). Our results support the conclusion that colostrum contains more OS compared with milk from the days following parturition. Collection of milk samples from a wider range of lactation stages is underway to investigate variations even in later lactation stages.

\section{Variation Between Jersey and Holstein Cows}

Milk samples at 3 time points (colostrum, d 6, and d 120) from 4 Jersey cows (cows 630, 632, 636, and 643) were selected for the breed variation study. Figure 6 shows extracted ion chromatograms of ion $\mathrm{m} / \mathrm{z} 636$ representing sialyllactose. The intensity varied from $0.9 \times$ $10^{6}$ to $1.5 \times 10^{6}$ in colostrum, whereas variations were fewer in transitional and mature milk. There are slight decreases in concentration of sialyllactose between d 6 and d 120 milk, except for cow 632, in which the bMO 


\begin{tabular}{|c|c|c|c|c|c|c|c|c|c|c|c|c|c|}
\hline \multirow[b]{2}{*}{ OS } & \multirow[b]{2}{*}{$m / z$ (expt) } & \multirow[b]{2}{*}{$m / z(\mathrm{cal})$} & \multirow[b]{2}{*}{$m / z$ (delta) } & \multirow[b]{2}{*}{ Hex } & \multirow[b]{2}{*}{ HexNAc } & \multirow[b]{2}{*}{$\mathrm{NeuAc}$} & \multirow[b]{2}{*}{ NeuGc } & \multicolumn{2}{|c|}{ Colostrum } & \multicolumn{2}{|c|}{ Day 6 milk } & \multicolumn{2}{|c|}{ Day 120 milk } \\
\hline & & & & & & & & RT (min) & $\mathrm{Ab}(\%)$ & $\mathrm{RT}(\min )$ & $\mathrm{Ab}(\%)$ & $\mathrm{RT}(\min )$ & $\mathrm{Ab}(\%)$ \\
\hline 1 & 506.1839 & 506.1846 & -0.0007 & 3 & & & & 10.160 & 0.11 & 10.135 & 0.27 & 10.203 & 0.46 \\
\hline 2 & 506.1833 & 506.1846 & -0.0013 & 3 & & & & 14.762 & 2.27 & 14.645 & 10.21 & 14.864 & 18.70 \\
\hline 3 & 547.2110 & 547.2112 & -0.0002 & 2 & 1 & & & 9.287 & 0.11 & 9.312 & 0.36 & 9.362 & 1.59 \\
\hline 4 & 547.2098 & 547.2112 & -0.0014 & 2 & 1 & & & 15.221 & 2.51 & 15.137 & 1.58 & 15.221 & 0.00 \\
\hline 5 & 709.2621 & 709.2640 & -0.0019 & 3 & 1 & & & 15.349 & 4.15 & 15.260 & 15.33 & 15.441 & 10.00 \\
\hline 6 & 750.2902 & 750.2906 & -0.0004 & 2 & 2 & & & 13.503 & 1.35 & 13.350 & 7.51 & 13.614 & 0.62 \\
\hline 7 & 750.2892 & 750.2906 & -0.0014 & 2 & 2 & & & 14.437 & 5.53 & 14.339 & 3.57 & 14.339 & 0.00 \\
\hline 8 & 871.3153 & 871.3168 & -0.0015 & 4 & 1 & & & 16.272 & 7.35 & 16.176 & 15.21 & 16.373 & 12.46 \\
\hline 9 & 912.3419 & 912.3434 & -0.0015 & 3 & 2 & & & 13.842 & 1.18 & 13.729 & 0.85 & 13.978 & 0.17 \\
\hline 10 & 912.3409 & 912.3434 & -0.0025 & 3 & 2 & & & 14.848 & 4.73 & 14.775 & 5.02 & 14.944 & 2.85 \\
\hline 11 & 1074.3977 & 1074.3962 & 0.0015 & 4 & 2 & & & 19.580 & 2.04 & 19.524 & 2.15 & 19.664 & 0.52 \\
\hline 12 & 635.2276 & 635.2272 & 0.0004 & 2 & & 1 & & 13.011 & 0.58 & 12.794 & 1.85 & 12.772 & 0.33 \\
\hline 13 & 635.2263 & 635.2272 & -0.0009 & 2 & & 1 & & 20.545 & 29.35 & 21.278 & 17.95 & 21.039 & 22.61 \\
\hline 14 & 676.2533 & 676.2538 & -0.0005 & 1 & 1 & 1 & & 12.759 & 1.52 & 12.533 & 0.82 & 14.814 & 1.01 \\
\hline 15 & 676.2523 & 676.2538 & -0.0015 & 1 & 1 & 1 & & 15.143 & 2.95 & 15.006 & 0.85 & 15.006 & 0.00 \\
\hline 16 & 676.2511 & 676.2538 & -0.0027 & 1 & 1 & 1 & & 22.021 & 1.00 & 21.980 & 0.64 & 21.980 & 0.00 \\
\hline 17 & 797.2782 & 797.2800 & -0.0018 & 3 & & 1 & & 21.823 & 0.17 & 21.823 & 0.00 & 21.294 & 3.28 \\
\hline 18 & 797.2772 & 797.2800 & -0.0028 & 3 & & 1 & & 24.639 & 4.98 & 24.530 & 3.74 & 24.257 & 23.11 \\
\hline 19 & 838.3056 & 838.3066 & -0.0010 & 2 & 1 & 1 & & 13.809 & 3.34 & 13.650 & 3.50 & 13.654 & 0.61 \\
\hline 20 & 838.3043 & 838.3066 & -0.0023 & 2 & 1 & 1 & & 15.380 & 0.75 & 15.193 & 0.27 & 16.783 & 0.31 \\
\hline 21 & 1000.3564 & 1000.3594 & -0.0030 & 3 & 1 & 1 & & 20.134 & 0.01 & 20.641 & 0.65 & 20.255 & 0.42 \\
\hline 22 & 1000.3554 & 1000.3594 & -0.0040 & 3 & 1 & 1 & & 23.717 & 0.58 & 23.569 & 0.41 & 23.530 & 0.42 \\
\hline 23 & 1000.3562 & 1000.3594 & -0.0032 & 3 & 1 & 1 & & 27.109 & 0.23 & 27.027 & 0.26 & 27.027 & 0.00 \\
\hline 24 & 1041.3862 & 1041.3860 & 0.0002 & 2 & 2 & 1 & & 21.835 & 2.29 & 21.796 & 1.17 & 21.796 & 0.00 \\
\hline 25 & 1162.4223 & 1162.4122 & 0.0101 & 4 & 1 & 1 & & 23.165 & 0.78 & 23.012 & 0.76 & 22.985 & 0.26 \\
\hline 26 & 1162.4109 & 1162.4122 & -0.0013 & 4 & 1 & 1 & & 20.785 & 0.50 & 20.711 & 0.19 & 20.711 & 0.00 \\
\hline 27 & 1203.4407 & 1203.4388 & 0.0019 & 3 & 2 & 1 & & 24.790 & 0.72 & 24.692 & 0.14 & 24.692 & 0.00 \\
\hline 28 & 1203.4380 & 1203.4388 & -0.0008 & 3 & 2 & 1 & & 26.494 & 0.69 & 26.359 & 0.20 & 26.359 & 0.00 \\
\hline 29 & 1203.4376 & 1203.4388 & -0.0012 & 3 & 2 & 1 & & 26.004 & 2.75 & 25.915 & 0.92 & 25.915 & 0.00 \\
\hline 30 & 1365.4909 & 1365.4916 & -0.0007 & 4 & 2 & 1 & & 20.990 & 0.11 & 22.117 & 0.08 & 22.117 & 0.00 \\
\hline 31 & 926.3209 & 926.3226 & -0.0017 & 2 & & 2 & & 25.144 & 0.45 & 25.144 & 0.00 & 25.144 & 0.00 \\
\hline 32 & 967.3499 & 967.3492 & 0.0007 & 1 & 1 & 2 & & 25.199 & 0.25 & 25.199 & 0.00 & 25.199 & 0.00 \\
\hline 33 & 967.3455 & 967.3492 & -0.0037 & 1 & 1 & 2 & & 21.314 & 0.03 & 21.314 & 0.00 & 21.314 & 0.00 \\
\hline 34 & 942.3234 & 942.3175 & 0.0059 & 2 & & 1 & 1 & 20.007 & 11.48 & 20.613 & 2.24 & 20.613 & 0.00 \\
\hline 35 & 651.2211 & 651.2221 & -0.0010 & 2 & & & 1 & 14.540 & 0.15 & 14.540 & 0.00 & 14.540 & 0.00 \\
\hline 36 & 692.2513 & 692.2487 & 0.0026 & 1 & 1 & & 1 & 20.237 & 0.15 & 20.237 & 0.00 & 20.237 & 0.00 \\
\hline 37 & 692.2464 & 692.2487 & -0.0023 & 1 & 1 & & 1 & 24.130 & 0.23 & 24.130 & 0.00 & 24.130 & 0.00 \\
\hline 38 & 813.2723 & 813.2749 & -0.0026 & 3 & & & 1 & 19.830 & 0.02 & 19.830 & 0.00 & 19.830 & 0.00 \\
\hline 39 & 1016.3604 & 1016.3543 & 0.0061 & 3 & 1 & & 1 & 23.082 & 0.09 & 22.924 & 0.07 & 22.924 & 0.00 \\
\hline 40 & 1178.4059 & 1178.4071 & -0.0012 & 4 & 1 & & 1 & 27.174 & 2.53 & 27.048 & 1.25 & 27.129 & 0.26 \\
\hline
\end{tabular}

${ }^{1}$ Expt and cal $=$ experimental and calculated $m / z$ values; delta $=$ expt - cal; Hex $=$ hexose; HexNAc $=N$-acetylhexosamine; NeuAc $=N$-acetyl neuraminic acid; NeuGc $=$ $N$-glycolylneuraminic acid. 


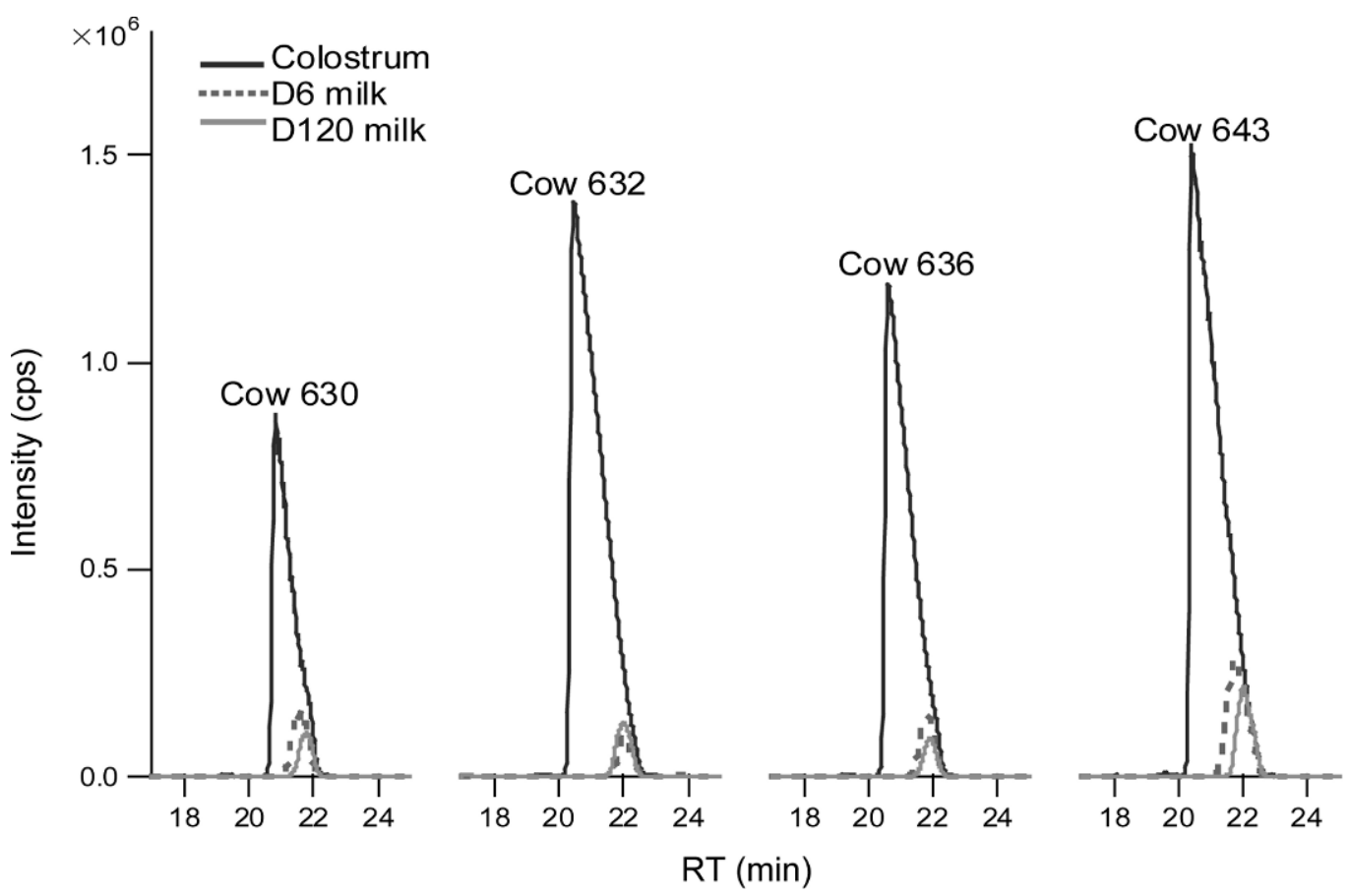

Figure 6. Extracted ion chromatograms of ion $m / z 636.2346$ from 4 Jersey cows at different lactation points. RT $=$ retention time.

concentration at d 120 is slightly higher than that at d 6 .

Figure 7 shows variation in the total OS abundance of Jersey cows during the different lactation stages; the same trend was found in Holsteins. The colostrum has much higher in concentration of sialyllactose, and decreased dramatically in d 6 milk, and decreased further in mature milk (d 120). Sialylation is surprisingly high in colostrum $(>80 \%)$ but decreased to around $50 \%$ in d 6 milk and to approximately $40 \%$ in d 120 milk. From these data, sialic OS represent 70 to $80 \%$ of total OS in colostrum from Holstein cows, decreasing to 50 to $60 \%$ in d 6 and d 120 milk samples. Both breeds show the same pattern, decreasing both in OS and sialylation concentration. Our results do not indicate significant differences between the breeds as previously reported (McJarrow and van Amelsfort-Schoonbeek, 2004). However, a larger sample pool of milk from various breeds should be studied before drawing definitive conclusions.

\section{DISCUSSION}

A comparison between $\mathrm{hMO}$ and bMO is appropriate given that so much more is known about hMO. In a previous study performed in our laboratory, we found unique differences between bMO and hMO in terms of the size, type, and relative amounts. Human milk oligosaccharides are highly fucosylated, with fucosylated OS accounting for up to $70 \%$ of OS species. In contrast, no fucosylated OS were found in bovine milk. Anionic OS are minor components in hMO $(<20 \%)$, but represent about $70 \%$ of the total OS in bovine colostrum. In addition, less complex structures with fewer isomers were found in bMO compared with hMO.

There is a paucity of data regarding the change in specific OS over the lactation period. For the most part, bulk variations in abundance in the early stages of lactation have been reported. For example, concentrations of hMO of 20 to $23 \mathrm{~g} / \mathrm{L}$ in colostrum decrease to 12 to $14 \mathrm{~g} / \mathrm{L}$ in mature milk (Coppa et al., 1999). Bovine milk sialylated oligosaccharides decrease dramatically during the first $24 \mathrm{~h}$ of lactation, whereas neutral OS increase (Nakamura et al., 2003). Other studies report an increasing mode of anionic OS in late lactation (Martin et al., 2001). Total OS concentration decreased along with anionic OS, which accounted for $70 \%$ of bMO.

It is now known that milk OS have at least 2 important potential functions: prevention of pathogen binding to the intestinal epithelial and stimulation of growth of beneficial bacterial (Newburg, 2000; Newburg et al., 2005). Both fucosylation and sialylation play a significant role in both functions. However, hMO are highly fucosylated, whereas bMO are not fucosylated. There have been reports of fucosylated bMO (Saito et al., 1987), but given the results presented herein, we question those findings. In the earlier publication, only 
A

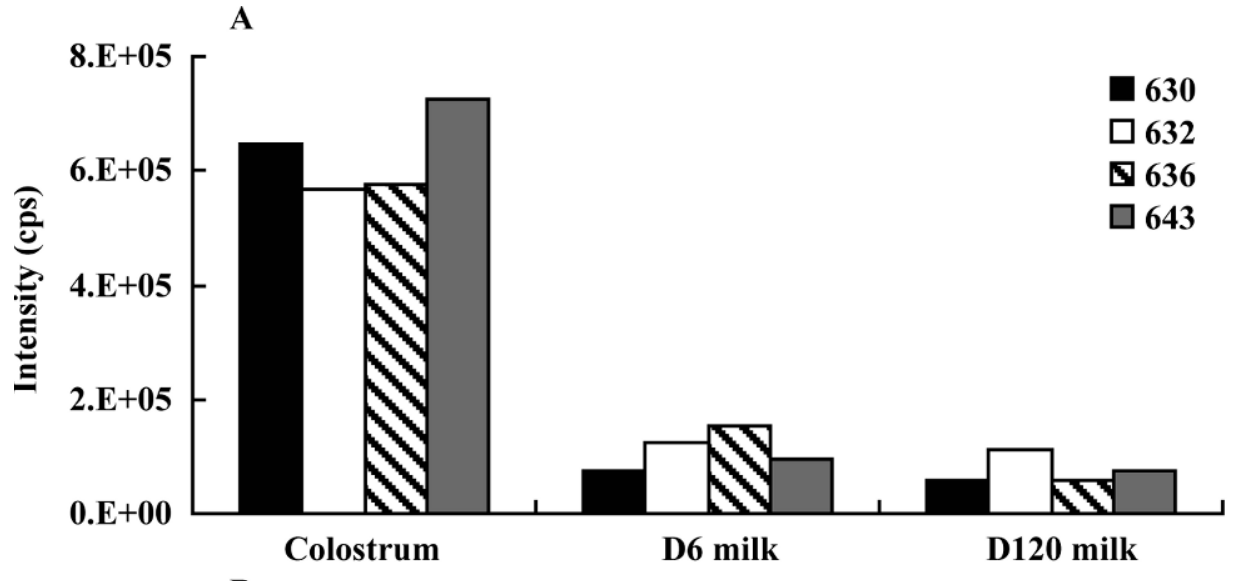

B

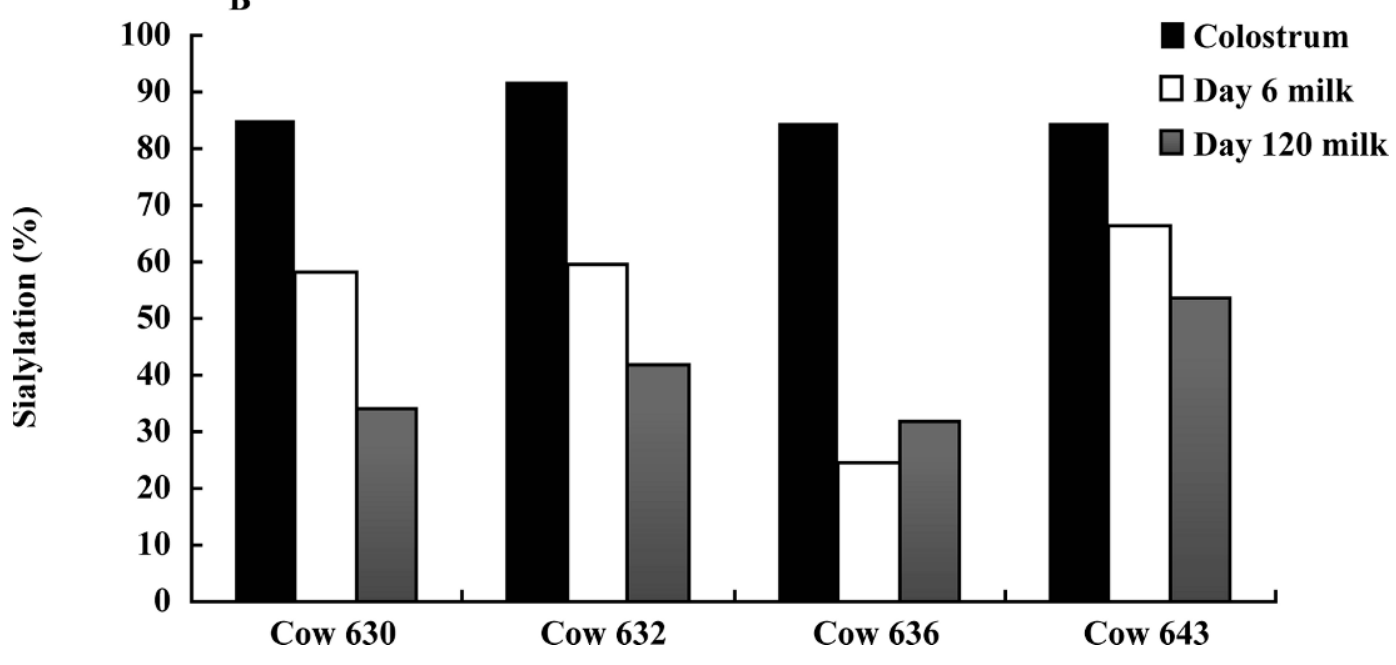

Figure 7. Changes in the total oligosaccharide intensity (A) and sialylation percentage (B) of Jersey cows (cows $630,632,636$, and 643$)$ at different stages of lactation (colostrum, d 6, and d 120 representing colostrum, transitional, and mature milk, respectively).

3 OS were observed, of which 1 was fucosylated. With a much more sensitive technique, we found up to $40 \mathrm{OS}$, none of which were fucosylated.

Sialylated OS also have important functions, particularly in brain development in infants (Wang and BrandMiller, 2003; Boehm and Stahl, 2007). In our study, sialylated OS make up $70 \%$ of colostrum and $50 \%$ of mature milk, and can be very useful and important components in the infant formula industry. The majority of the sialic acid is NeuAc (around 93\%) with the remainder NeuGc (around 7\%) in colostrum. However, for mature milk, NeuGc decreases to less than $4 \%$ of the sialic acid in d 6 milk and to $0 \%$ in mature milk samples.

Furthermore, we chose Jersey cow for the breed variation study. In both Jersey and Holstein milk, the total OS concentration decreased dramatically in transitional milk (d 6) and decreased slightly in mature milk (d 120). There is variation among individuals, but the differences are smaller in later lactation samples.
Compared with Holstein milk, Jersey milk has more anionic components and a higher concentration of bMO.

By using the HPLC-chip/TOF MS system, we were able to monitor each OS species consistently and accurately, providing a complete picture of OS variation during lactation. The excellent reproducibility of the microfluidic chip was also demonstrated.

\section{ACKNOWLEDGMENTS}

Funding provided by the California Dairy Research Foundation (Davis, CA), Dairy Management Inc. (Rosemont, IL), the University of California Discovery (Berkeley), and the National Institutes of Health (Bethesda, MD) is gratefully acknowledged.

\section{REFERENCES}

Ali-Vehmas, T., M. Vikerpuur, S. Pyorala, and F. Atroshi. 2001. Characterization of hemolytic activity of Staphylococcus aureus 
strains isolated from bovine mastitic milk. Microbiol. Res. 155:339-344.

Boehm, G., and B. Stahl. 2007. Oligosaccharides from milk. J. Nutr. 137(Suppl. 2):847S-849S

Boehm, G., B. Stahl, J. Jelinek, J. Knol, V. Miniello, and G. E. Moro. 2005. Prebiotic carbohydrates in human milk and formulas. Acta Paediatr. Suppl. 94:18-21.

Bongers, M. E., F. de Lorijn, J. B. Reitsma, M. Groeneweg, J. A. Taminiau, and M. A. Benninga. 2007. The clinical effect of a new infant formula in term infants with constipation: A double-blind, randomized cross-over trial. Nutr. J. 6:8.

Brunser, O., G. Figueroa, M. Gotteland, E. Haschke-Becher, C. Magliola, F. Rochat, S. Cruchet, R. Palframan, G. Gibson, F. Chauffard, and F. Haschke. 2006. Effects of probiotic or prebiotic supplemented milk formulas on fecal microbiota composition of infants. Asia Pac. J. Clin. Nutr. 15:368-376.

Chaturvedi, P., C. D. Warren, M. Altaye, A. L. Morrow, G. RuizPalacios, L. K. Pickering, and D. S. Newburg. 2001. Fucosylated human milk oligosaccharides vary between individuals and over the course of lactation. Glycobiology 11:365-372.

Coppa, G. V., P. Pierani, L. Zampini, S. Bruni, I. Carloni, and O. Gabrielli. 2001. Characterization of oligosaccharides in milk and feces of breast-fed infants by high-performance anion-exchange chromatography. Adv. Exp. Med. Biol. 501:307-314.

Coppa, G. V., P. Pierani, L. Zampini, I. Carloni, A. Carlucci, and O. Gabrielli. 1999. Oligosaccharides in human milk during different phases of lactation. Acta Paediatr. Suppl. 88:89-94.

German, J. B., S. L. Freeman, C. B. Lebrilla, and D. A. Mills. 2008. Human milk oligosaccharides: evolution, structures and bioselectivity as substrates for intestinal bacteria. Nestle Nutr. Workshop Ser. Pediatr. Program. 62:205-218, discussion 218 222.

Gopal, P. K., and H. S. Gill. 2000. Oligosaccharides and glycoconjugates in bovine milk and colostrum. Br. J. Nutr. 84(Suppl. 1):S69 S74.

Guerardel, Y., W. Morelle, Y. Plancke, J. Lemoine, and G. Strecker. 1999. Structural analysis of three sulfated oligosaccharides isolated from human milk. Carbohydr. Res. 320:230-238.

Jackson, J. R., W. L. Hurley, R. A. Easter, A. H. Jensen, and J. Odle. 1995. Effects of induced or delayed parturition and supplemental dietary fat on colostrum and milk composition in sows. J. Anim. Sci. 73:1906-1913.

Kunz, C., and S. Rudloff. 1996. Structural and functional aspects of oligosaccharides in human milk. Z. Ernahrungswiss. 35:22-31. (in German)

Kunz, C., S. Rudloff, W. Baier, N. Klein, and S. Strobel. 2000. Oligosaccharides in human milk: Structural, functional, and metabolic aspects. Annu. Rev. Nutr. 20:699-722.

Martin, M. J., S. Martin-Sosa, L. A. Garcia-Pardo, and P. Hueso. 2001. Distribution of bovine milk sialoglycoconjugates during lactation. J. Dairy Sci. 84:995-1000.

Martin-Sosa, S., M. J. Martin, L. A. Garcia-Pardo, and P. Hueso. 2003. Sialyloligosaccharides in human and bovine milk and in infant formulas: Variations with the progression of lactation. J. Dairy Sci. 86:52-59.

McJarrow, P., and J. van Amelsfort-Schoonbeek. 2004. Bovine sialyl oligosaccharides: Seasonal variations in their concentrations in milk, and a comparison of the colostrums of Jersey and Friesian cows. Int. Dairy J. 14:571-579.

Moughan, P. J., M. J. Birtles, P. D. Cranwell, W. C. Smith, and M. Pedraza. 1992. The piglet as a model animal for studying aspects of digestion and absorption in milk-fed human infants. World Rev. Nutr. Diet. 67:40-113.
Nakamura, T., H. Kawase, K. Kimura, Y. Watanabe, M. Ohtani, I. Arai, and T. Urashima. 2003. Concentrations of sialyloligosaccharides in bovine colostrum and milk during the prepartum and early lactation. J. Dairy Sci. 86:1315-1320.

Newburg, D. S. 1996. Oligosaccharides and glycoconjugates in human milk: Their role in host defense. J. Mammary Gland Biol. Neoplasia 1:271-283.

Newburg, D. S. 2000. Oligosaccharides in human milk and bacterial colonization. J. Pediatr. Gastroenterol. Nutr. 30(Suppl. 2):S8S17.

Newburg, D. S., G. M. Ruiz-Palacios, M. Altaye, P. Chaturvedi, M. L. Guerrero, J. K. Meinzen-Derr, and A. L. Morrow. 2004. Human milk alpha 1,2-linked fucosylated oligosaccharides decrease risk of diarrhea due to stable toxin of $E$. coli in breastfed infants. Adv. Exp. Med. Biol. 554:457-461.

Newburg, D. S., G. M. Ruiz-Palacios, and A. L. Morrow. 2005. Human milk glycans protect infants against enteric pathogens. Annu. Rev. Nutr. 25:37-58.

Ninonuevo, M. R., Y. Park, H. Yin, J. Zhang, R. E. Ward, B. H. Clowers, J. B. German, S. L. Freeman, K. Killeen, R. Grimm, and C. B. Lebrilla. 2006. A strategy for annotating the human milk glycome. J. Agric. Food Chem. 54:7471-7480.

Ninonuevo, M. R., P. D. Perkins, J. Francis, L. M. Lamotte, R. G. LoCascio, S. L. Freeman, D. A. Mills, J. B. German, R. Grimm, and C. B. Lebrilla. 2008. Daily variations in oligosaccharides of human milk determined by microfluidic chips and mass spectrometry. J. Agric. Food Chem. 56:618-626.

Pfenninger, A., M. Karas, B. Finke, and B. Stahl. 2002. Structural analysis of underivatized neutral human milk oligosaccharides in the negative ion mode by nano-electrospray MS(n) (part 2: application to isomeric mixtures). J. Am. Soc. Mass Spectrom. 13:1341-1348.

Pfenninger, A., M. Karas, B. Finke, B. Stahl, and G. Sawatzki. 2001. Mass spectrometric investigations of human milk oligosaccharides. Adv. Exp. Med. Biol. 501:279-284.

Saito, T. T. Itoh and S. Adachi. 1987. Chemical structure of three neutral trisaccharides isolated in free form from bovine colostrum. Carbohydr. Res. 165:43-51.

Suzuki, M., and A. Suzuki. 2001. Structural characterization of fucose-containing oligosaccharides by high-performance liquid chromatography and matrix-assisted laser desorption/ionization time-of-flight mass spectrometry. Biol. Chem. 382:251-257.

Tao, N., E. J. DePeters, S. Freeman, J. B. German, R. Grimm, and C. B. Lebrilla. 2008. Bovine milk glycome. J. Dairy Sci. 91:37683778 .

Urashima, T., T. Saito, T. Nakamura, and M. Messer. 2001. Oligosaccharides of milk and colostrum in non-human mammals. Glycoconj. J. 18:357-371.

Wang, B., and J. Brand-Miller. 2003. The role and potential of sialic acid in human nutrition. Eur. J. Clin. Nutr. 57:1351-1369.

Wang, B., B. Yu, M. Karim, H. Hu, Y. Sun, P. McGreevy, P. Petocz, S. Held, and J. Brand-Miller. 2007. Dietary sialic acid supplementation improves learning and memory in piglets. Am. J. Clin. Nutr. 85:561-569.

Ward, R. E., M. Ninonuevo, D. A. Mills, C. B. Lebrilla, and J. B. German. 2006. In vitro fermentation of breast milk oligosaccharides by Bifidobacterium infantis and Lactobacillus gasseri. Appl. Environ. Microbiol. 72:4497-4499.

Wilson, N. L., L. J. Robinson, A. Donnet, L. Bovetto, N. H. Packer, and N. G. Karlsson. 2008. Glycoproteomics of milk: Differences in sugar epitopes on human and bovine milk fat globule membranes. J. Proteome Res. 7:3687-3696. 